Ergod. Th. \& Dynam. Sys. (1983), 3, 567-578

Printed in Great Britain

\title{
Persistence in expansive systems
}

\author{
JORGE LEWOWICZ \\ Departamento de Matemáticas, Universidad Simón Bolívar, Apartado Postal 80.659. \\ Caracas, Venezuela
}

(Received 20 July 1982)

\begin{abstract}
We give some sufficient conditions for an expansive diffeomorphism $f$ of a compact manifold to be such that every neighbouring diffeomorphism shows, roughly, all the dynamical features of $f$. These results are then applied to prove a structural stability theorem for pseudo-Anosov maps.
\end{abstract}

\section{0 . Introduction}

Let $f$ be a homeomorphism of the compact riemannian manifold $M$; we say that the trajectory through $x \in M$ is persistent if given $\varepsilon>0$ there exists a $C^{0}$-neighbourhood of $f$ such that if $g$ belongs to that neighbourhood, then for some $y \in M$,

$$
\operatorname{dist}\left(f^{n}(x), g^{n}(y)\right)<\varepsilon, \quad n \in \mathbb{Z} .
$$

In this paper we give some sufficient conditions for this property to hold uniformly on certain $f$-invariant subsets of $M$, and, as an application, we prove that Thurston's pseudo-Anosov maps are 'structurally stable'.

When $f$ is expansive and $M$ itself is (uniformly) persistent then, roughly, the dynamics of $f$ may be found in each $g$ close enough to $f$ in the $C^{0}$-topology (see $\S 1$ ); however, these $g$ may present dynamical features with no counterpart in $f$. Thus, (uniform) persistence of $M$ is a weaker property than topological stability ([7], [3]); nevertheless, if we restrict the perturbations to a suitable class, we may still get conjugacy between $f$ and $g$.

Among other results we prove, for expansive $f$, that if $M$ is a two or threedimensional manifold, the set of non-wandering points of $f$ is persistent (see $\S 1$ for the definition) provided it contains a dense subset of hyperbolic periodic points. We also show that in the case where $f$ preserves a smooth volume form, then Pesin's region (see $[5, \S 1.7]$ ) is persistent when it has positive measure (theorems $2.8,2.9$ ). For pseudo-Anosov $f$ we show that if $g$ is $C^{1}$-close enough to $f$ and coincides with $f$ at singular points, then $g$ is conjugate to $f$ (theorem 3.5).

We believe that, apart from such applications, there is another reason for studying these persistence properties: it seems plausible to think that if a theory of asymptotic behaviour is possible, then semi-persistence (i.e. persistence of positive or negative semi-trajectories) should hold on big subsets of $M$ for large classes of dynamical systems. For recurrent trajectories (the case we shall mainly be concerned with) both notions, persistence and semi-persistence, are equivalent, as may easily be shown. 
Finally, I would like to thank M. Gerber and A. Katok for useful conversations on these topics.

\section{Persistence}

Let $M$ be a compact connected riemannian smooth manifold and $f: M \rightarrow M$ a homeomorphism. An $f$-invariant subset $K \subset M$ is persistent if for each $\varepsilon>0$ there exists a $C^{0}$-neighbourhood $N=N(K, \varepsilon)$ of $f$ such that for each $x \in K^{\dagger}$ and each $g \in N$ there exists $y \in M$ such that

$$
\operatorname{dist}\left(f^{n}(x), g^{n}(y)\right)<\varepsilon \quad \text { for every } n \in \mathbb{Z} \text {. }
$$

When $K=M(K=\Omega(f))$ we say that $f$ is persistent (resp. $\Omega$-persistent). Topologically stable homeomorphisms are persistent but, as may easily be shown, there are persistent homeomorphisms, for instance pseudo-Anosov maps, that are not topologically stable.

Obviously if $K$ is persistent, so is its closure $\bar{K}$; we shall therefore consider only compact subsets of $M$. Assume now that $f$ is expansive, i.e. that there exists $\alpha>0$ such that if dist $\left(f^{n}(x), f^{n}(y)\right) \leq \alpha$ for every $n \in \mathbb{Z}$, then $x=y$. Such an $\alpha$ is called an expansivity constant of $f$. The following lemma states essentially that except for an identification of 'indistinguishable' points, dynamical systems with expansivity constant $\alpha$ form an open set in the $C^{0}$-topology.

LEMMA 1.1. For every $\delta>0$, there exists a $C^{0}$-neighbourhood $N$ of $f$ such that if $x$, $y \in M, g \in N$ and dist $\left(g^{n}(x), g^{n}(y)\right) \leq \alpha, n \in \mathbb{Z}$, then dist $\left(g^{n}(x), g^{n}(y)\right) \leq \delta, n \in \mathbb{Z}$.

Proof. Since $M$ is compact and $f$ is expansive it is easy to show that there exists $m \in \mathbb{Z}^{+}$such that dist $(x, y) \geq \delta$ implies dist $\left(f^{n}(x), f^{n}(y)\right)>\alpha$ for some $n \in \mathbb{Z},|n| \leq m$. If

$$
\mu=\min _{x, y \in M, \operatorname{dist}(x, y) \geq \delta} \max _{|n| \leq m} \operatorname{dist}\left(f^{n}(x), f^{n}(y)\right),
$$

then $\mu>\alpha$. Let $N$ be chosen in such a way that for $g$ in $N$,

$$
\operatorname{dist}\left(g^{n}(x), f^{n}(x)\right)<\frac{1}{2}(\mu-\alpha) \quad \text { for every } x \in M,|n| \leq m \text {. }
$$

Then if $g \in N, x, y \in M$, dist $\left(g^{n}(x), g^{n}(y)\right) \leq \alpha$ for $n \in \mathbb{Z}$, and if for some $n_{0}$, dist $\left(g^{n_{0}}(x), g^{n_{0}}(y)\right) \geq \delta$, we have that for $|n| \leq m$,

$$
\begin{aligned}
\operatorname{dist} & \left(f^{n}\left(g^{n_{0}}(x)\right), f^{n}\left(g^{n_{0}}(y)\right)\right) \leq \operatorname{dist}\left(g^{n_{0}+n}(x), g^{n_{0}+n}(y)\right) \\
& +\operatorname{dist}\left(f^{n}\left(g^{n_{0}}(x)\right), g^{n+n_{0}}(x)\right)+\operatorname{dist}\left(f^{n}\left(g^{n_{0}}(y)\right), g^{n+n_{0}}(y)\right)<\mu,
\end{aligned}
$$

which is absurd. Consequently, dist $\left(g^{n}(x), g^{n}(y)\right) \leq \delta$ for every $n \in \mathbb{Z}$.

Choose $\delta \leq \frac{1}{2} \alpha$ and let $N=N(\delta)$ be the corresponding neighbourhood of $f$ given by lemma 1.1. For $g \in N$ define the relation $R_{g}$ by

$$
R_{\mathrm{g}}=\left\{(x, y) \in M \times M: \operatorname{dist}\left(g^{n}(x), g^{n}(y)\right) \leq \delta, n \in \mathbb{Z}\right\} ;
$$

then $R_{g}$ is an equivalence relation and each equivalence class is compact. If $\pi: M \rightarrow$ $M / R_{\mathrm{g}}$ is the canonical projection, we define a homeomorphism $\hat{g}$ of the compact Hausdorff space $M / R_{g}$ onto itself by

$$
\hat{g}(\pi(x))=\pi(g(x)), \quad x \in M \text {. }
$$


Suppose now that $K$ is a compact $f$-invariant subset of $M$ that is persistent; choose $\varepsilon<\frac{1}{2} \delta$ and let $N(K, \varepsilon)$ be the $C^{0}$-neighbourhood of $f$ mentioned in the definition of persistence.

LEMMA 1.2. There is a $C^{0}$-neighbourhood $N \subset N(\delta)$ of $f$ such that if $g \in N$, then there exists a compact $g$-invariant subset $K_{g}$ of $M$ such that $f / K$ and $\hat{g} / \pi\left(K_{g}\right)$ are conjugate.

Proof. Let $N=N(\delta) \cap N(K, \varepsilon)$ and

$$
K_{g}=\left\{y \in M: \operatorname{dist}\left(f^{n}(x), g^{n}(y)\right) \leq \varepsilon, n \in \mathbb{Z}, \text { for some } x \in K\right\} .
$$

If $y_{i} \in K_{g}, i=1,2$, and dist $\left(f^{n}(x), g^{n}\left(y_{i}\right)\right) \leq \varepsilon, n \in \mathbb{Z}$, then $\left(y_{1}, y_{2}\right) \in R_{g}$. Also, if for $i=1,2$, dist $\left(f^{n}\left(x_{i}\right), g^{n}\left(y_{i}\right)\right) \leq \varepsilon, n \in \mathbb{Z}$ and $\pi\left(y_{1}\right)=\pi\left(y_{2}\right)$, then dist $\left(f^{n}\left(x_{1}\right), f^{n}\left(x_{2}\right)\right) \leq$ $\alpha$ for $n \in \mathbb{Z}$, and therefore $x_{1}=x_{2}$. These remarks imply that the mapping $h: x \rightarrow \pi(y)$, where dist $\left(f^{n}(x), g^{n}(y)\right) \leq \varepsilon, n \in \mathbb{Z}$, is well defined and moreover, that $h: K \rightarrow \pi\left(K_{g}\right)$ is bijective. Since $h$ is continuous and $h(f(x))=\hat{g}(h(x)), h$ is a conjugacy between $f / K$ and $\hat{g} / \pi\left(K_{g}\right)$ as we had to show.

Remark. If $g$ is itself expansive, say with expansivity constant $\beta$, and lies in $N(\delta) \cap$ $N(K, \varepsilon)$, where $\delta$ and $\varepsilon$ are chosen as before, and in addition $\delta<\beta$, then $\pi / K_{\mathrm{g}}$ is a homeomorphism and $f / K$ is conjugate to $g / K_{\mathrm{g}}$. In particular, when $K=M$ we also have that $K_{\mathrm{g}}=M$ since $K_{\mathrm{g}}$ is open in $M$; thus, in this case $f$ is conjugate to $g$.

\section{Expansive systems}

Let $f$ be an expansive $C^{1}$-diffeomorphism of the compact, connected, smooth, riemannian manifold $M$, let $\beta>0$ be an expansivity constant for $f$ and let $P \subset M$ be $f$-invariant and such that for each $x \in P, x \in \alpha(x)$ and $x \in \omega(x)$, where $\alpha(x)$ and $\omega(x)$ denote the limit sets of the trajectory through $x$. Assume, furthermore, that at each $x \in P$ there are transversal local stable and unstable manifolds $S_{x}, U_{x}$. In other words, we assume that for each $x \in P$, there exists $g_{x}: B_{k} \rightarrow M, g_{x}(0)=x$, and $h_{x}: B_{l} \rightarrow M, h_{x}(0)=x,\left(B_{k}, B_{l}\right.$ denote respectively the unit balls in $R^{k}, R^{l}, k+l=$ $\operatorname{dim} M)$, such that $g_{x}$ and $h_{x}$ are $C^{1}$-embeddings, $g_{x}\left(B_{k}\right)$ 历 $h_{x}\left(B_{l}\right)$ and that for each $x \in P$, if $y \in S_{x}$ (resp. $\left.U_{x}\right)$, then

$$
\operatorname{dist}\left(f^{n}(x), f^{n}(y)\right)<\beta \quad \text { for } n \geq 0(\text { resp, } n \leq 0) \text {. }
$$

Here we have set $g_{x}\left(B_{k}\right)=S_{x}, h_{x}\left(B_{l}\right)=U_{x}$. We assume furthermore that for each $x \in P$ there exists $r_{x}>0$, such that if $y$ belongs to the trajectory through $x$ and dist $(x, y)<r_{x}$, then $S_{y} \pitchfork U_{x}$, and $S_{y} \cap U_{x} \neq \varnothing$.

Proposition 2.1. Suppose that for each $x \in P, S_{x}$ or $U_{x}$ is one-dimensional. Then $\bar{P}$ is persistent.

Proof. Let $(\hat{M}, \phi)$ be the suspension of $(M, f), \phi$ being the suspension flow; we assume that $\hat{M}$ is endowed with some riemannian metric. Call $p: M \times \mathbb{R} \rightarrow \hat{M}$ the covering projection and let $M_{t}$ stand for $p(M \times\{t\})$; we shall identify $M$ with $M_{0}$. It is easy to show that, under the assumptions of the proposition, if $\xi=p(x, t), x \in P$, there exist two cells transversely embedded in $M_{t}$, the local stable and unstable manifolds $S_{\xi}, U_{\xi}$ of $\xi$, that have with respect to $\phi$ the properties analogous to those 
which $S_{x}, U_{x}$ have with respect to $f$, as stated in the previous paragraph. Moreover if $\xi=p(x, 0)$, then $S_{\xi}, U_{\xi}$ coincide with $S_{x}, U_{x}$. Let

$$
A=\left\{(\xi, \eta) \in \hat{M} \times \hat{M}: \xi, \eta \in M_{t} \text { for some } t \in \mathbb{R}\right\}
$$

It follows from $[3, \S 4]$, that for some $\gamma>0$ there exists a Lyapunov function

$$
u:\{(\xi, \eta) \in A: \operatorname{dist}(\xi, \eta)<\gamma\} \rightarrow \mathbb{R}
$$

that vanishes on the diagonal of $\hat{M} \times \hat{M}$ and so that, if $0<\operatorname{dist}(\xi, \eta)<\gamma$, then

(i) $\mathcal{U}(\xi, \eta)>0$

(ii) $\dot{U}(\xi, \eta)<0(>0)$ for $\eta \in S_{\xi}$ (resp. $\eta \in U_{\xi}$ ),

(iii) $\ddot{\ddot{u}}(\xi, \eta)>0$.

Here $\dot{U}$ and $\ddot{\mathscr{U}}$ are defined as in [3, p. 197].

Let $\rho$ be a fixed positive number and choose a fixed $\sigma>0$ such that $\mathscr{U}(\xi, \eta)<\sigma$ implies dist $(\xi, \eta)<\rho$. Let $x \in P$ and let $g_{x}: B \rightarrow M$ be an embedding of the unit ball in $R^{m}$ (for suitable $m$ ) onto $S_{x}$. Let

$$
K_{\sigma}(x, t)=\left\{\eta \in M_{t}: \mathcal{U}\left(\phi_{t}(x), \eta\right) \leq \sigma\right\} .
$$

We assume, as we may, that $S_{x} \subset$ int $K_{\sigma}(x, 0)$ and prove the following lemma.

LEMMA 2.2. For each $y \in S_{x}, \phi(y, t) \in K_{\sigma}(x, t), t \geq 0$.

Proof. For $n$ large enough there is no $y \in S_{x}$ such that $\phi(y, t) \in K_{\sigma}(x, t), t \in[0, n]$ and $\phi(y, n) \in \partial K_{\sigma}(x, n)$. Otherwise we could find a sequence of integers $n_{k} \rightarrow \infty$ and a sequence of points $z_{n_{k}}, \mathscr{U}\left(f^{n_{k}}(x), z_{n_{k}}\right)=\sigma$, such that for each $k$,

$$
\operatorname{dist}\left(f^{n}\left(f^{n_{k}}(x)\right), f^{n}\left(z_{n_{k}}\right)\right) \leq \beta, \quad n \geq-n_{k} ;
$$

by taking limits, and since $f$ is expansive, we reach a contradiction. Now assume, arguing again by contradiction, that for some $t_{0}>0$ and some $y_{0} \in S_{x}$, we have that $\phi\left(y_{0}, t_{0}\right) \notin K_{\sigma}\left(x, t_{0}\right)$. Then, for some $n>0$, both sets

$$
\left\{y \in S_{x}: \phi(y, t) \notin K_{\sigma}(x, t) \text { for some } t, 0 \leq t \leq n\right\}
$$

and

$$
\left\{y \in S_{x}: \phi(y, t) \in \text { int } K_{\sigma}(x, t), 0 \leq t \leq n\right\}
$$

are open and non-empty. By (iii) and the assertion at the beginning of this proof, they are also disjoint; since obviously their union is the connected set $S_{x}$, this is absurd.

LEMMA 2.3. There exists $T=T(x)<0$, so that if $t \leq T$, then for any $u \in \partial B$, there exists $s=s(t, u), 0<s \leq 1$ such that $\phi(g(s u), t) \in \partial K_{\sigma}(x, t)$.

Proof. As before, the expansivity of $f$ implies that for each $u \in \partial B$ there exists $t_{0}<0$, such that $\phi\left(g(u), t_{0}\right) \notin K_{\sigma}\left(x, t_{0}\right)$. Then, if $t_{1}<t_{0}, \phi\left(g(s u), t_{1}\right) \in \partial K_{\sigma}\left(x, t_{1}\right)$ for some $s, 0<s \leq 1$, since otherwise, the set

$$
\left\{s \in[0,1]: \phi(g(s u), t) \notin K_{\sigma}(x, t) \text { for some } t \in\left[t_{1}, 0\right]\right\}
$$

would be non-empty, and then a connectedness argument like the previous one would lead to a contradiction. Since $\partial B$ is compact, this completes the proof of the lemma. 
We remark for further use that it follows, again by a connectedness argument, that if $t<0$ and $\phi(g(s u), t) \in K_{\sigma}(x, t)$ for $0 \leq s \leq s_{0}$, then

$$
\phi_{r}(\phi(g(s u), t)) \in K_{\sigma}(x, t+\tau)
$$

for any $\tau, 0 \leq \tau \leq-t$, and any $s, 0 \leq s \leq s_{0}$.

Choose $\rho^{\prime}>0$ such that $\mathcal{U}(\xi, \eta)<\sigma$ if $\xi, \eta \in A$ and dist $(\xi, \eta)<\rho^{\prime}$, and then choose $\sigma^{\prime}>0$, such that $U(\xi, \eta)<\sigma^{\prime}$ implies dist $(\xi, \eta)<\rho^{\prime}$. We assume with no loss of generality that the $T$ of lemma 2.3 is less than -1 and for $t \leq T$ we define $C_{t}$ by

$$
C_{t}=\left\{\phi(g(s u), t): 0 \leq s \leq s_{0}(t, u)\right\},
$$

where $\phi_{t}\left(g\left(s_{0}(t, u)\right) \in \partial K_{\sigma}(x, t)\right.$ and $\phi_{t}(g(s u)) \in$ int $K_{\sigma}(x, t)$ for $0 \leq s \leq s_{0}(t, u)$.

Let $\rho^{\prime \prime}>0$ be such that for $\xi, \eta \in A$, dist $(\xi, \eta) \leq \rho^{\prime \prime}$ implies $u(\xi, \eta)<\sigma^{\prime}$, and choose $l>0$ with the property that

$$
\operatorname{dist}\left(\phi_{t}(\xi), \phi_{t}(\eta)\right) \geq l \operatorname{dist}(\xi, \eta) \quad \text { for } 0 \leq t \leq 1 \quad \text { and } \xi, \eta \in \hat{M} \text {. }
$$

LEMMA 2.4. $\dot{\imath}\left(\phi_{t}(x), \eta\right)$ is bounded away from zero uniformly on

$$
\left\{\eta \in C_{t} \cap \partial K_{\sigma^{\prime}}(x, t): t \leq T, x \in P\right\} .
$$

Proof. Let $\mu=\min \ddot{u}(\xi, \eta)$ for $\xi, \eta \in A$ and $l \rho^{\prime \prime} \leq \operatorname{dist}(\xi, \eta) \leq \rho$. Then by the remark at the end of the last paragraph, we may write for $u \in \partial B, t \leq T$, and $0 \leq s \leq s_{0}(t, u)$,

$$
\begin{aligned}
\dot{u}(x, g(s u))-\dot{u}\left(\phi_{t}(x), \phi_{t}(g(s u))\right) & =\int_{t}^{0} \ddot{\mathscr{u}}\left(\phi_{\tau}(x), \phi_{\tau}(g(s u))\right) d \tau \\
& \geq \int_{t}^{t+1} \ddot{\ddot{u}}\left(\phi_{\tau}(x), \phi_{\tau}(g(s u))\right) d \tau \geq \mu .
\end{aligned}
$$

Since $\dot{U}(x, g(s u)) \leq 0$ (see [3, p. 202]), this incquality proves the lemma.

Now we assume that $U_{x}$ is one dimensional. Since the previous arguments also apply to $U_{x}$ when we move forward, for some $z=f^{n}(x), n>0$, there is an embedding $h_{z}:[-1,1] \rightarrow M, h_{z}(0)=z$ such that

$$
\begin{gathered}
h_{z}(s) \in K_{\sigma^{\prime}}(z, 0), \quad-1 \leq s \leq 1, \\
h_{z}(-1), h_{z}(1) \in \partial K_{\sigma^{\prime}}(z, 0)
\end{gathered}
$$

and

$$
\phi_{t}\left(h_{z}(s)\right) \in K_{\sigma^{\prime}}(z, t), \quad t \leq 0 \text {. }
$$

Moreover, the positive numbers $\dot{u}\left(z, h_{z}(-1)\right), \dot{U}\left(z, h_{z}(1)\right)$ are uniformly bounded away from zero for $x \in P$, by the forward version of lemma 2.4. As $f$ is expansive $h([-1,1]) \cap S_{z}=\{z\}$.

For a suitable sequence $t_{\nu} \rightarrow-\infty, \phi\left(x, t_{\nu}\right) \rightarrow z$ and if $\nu$ is large enough dist $\left(\phi\left(x, t_{v}\right), z\right)<r_{z}$. Therefore $C_{t, n} \cap h([-1,1]) \neq \varnothing$ and $C_{t_{t}} \pi h([-1,1])$. Since $\dot{u}(\xi, \eta)<0(>0)$ for any pair of points $\xi, \eta, \xi \neq \eta, \xi, \eta \in C_{t_{\nu}}$ (resp. $\left.h([-1,1])\right)$, then $C_{t_{\nu}}$ and $h([-1,1])$ have exactly one point of transversal intersection.

Let $s_{\nu} \in(-1,1)$ be such that $h\left(s_{\nu}\right) \in C_{t_{\nu}}$. It is easy to see that if $\nu$ is larger than some $\nu_{0}(x)$ we may find points $u_{\nu}, l_{\nu} \in \partial K_{\sigma^{\prime}}\left(x, t_{v}\right)$ such that 
(1) If $s>0$ is small enough, $u_{\nu}\left(l_{\nu}\right)$ may be joined, within the ball of radius $\rho^{\prime}$ centred at $\phi\left(x, t_{\nu}\right)$, to $h\left(s_{\nu}+s\right)$ by an arc transversal to $C_{t_{\nu}}$ whose $(\bmod 2)$ intersection number with $C_{t_{v}}$ is 0 (resp. 1 ).

(2) For some $\lambda>0$,

$$
\dot{U}\left(\phi\left(x, t_{\nu}\right), u_{\nu}\right), \dot{u}\left(\phi\left(x, t_{\nu}\right), l_{\nu}\right)>\lambda,
$$

for every $x \in P$.

(3) There is an arc $\chi_{\nu}$ joining $u_{\nu}$ to $l_{\nu}$ within $K_{\sigma^{\prime}}\left(x, t_{\nu}\right)$.

Let $\psi$ be a flow on $\hat{M}$ such that $\psi_{t^{\prime}}\left(M_{t}\right)=M_{t+t^{\prime}}$. If $\psi$ is $C^{1}$-close enough to $\phi$, we have

(a) ${ }^{m} \dot{u}(\xi, \eta)<0 \quad$ if $\xi=\phi(x, t)$ and $\eta \in C_{t} \cap \partial K_{\sigma^{\prime}}(x, t), t \leq T$

(b) ${ }^{m} \dot{U}\left(\xi, u_{\nu}\right), m \dot{U}\left(\xi, l_{\nu}\right)>0 \quad$ if $\xi=\phi\left(x, t_{\nu}\right)$ and $\nu>\nu_{0}(x)$.

(c) $m \ddot{u}(\xi, \eta)>0, \quad$ if $\eta \in \partial K_{\sigma^{\prime}}(x, t), t \leq T$.

Here

$$
m \dot{u}(\xi, \eta)=\lim _{t \rightarrow 0} 1 / t\left\{u\left(\phi_{t}(\xi), \psi_{t}(\eta)\right)-\mathcal{u}(\xi, \eta)\right\}
$$

and

$$
m \ddot{\mathscr{U}}(\xi, \eta)=\lim _{t \rightarrow 0} 1 / t\left\{{ }^{m} \dot{\mathscr{U}}\left(\phi_{t}(\xi), \psi_{t}(\eta)\right)-m \dot{\mathscr{U}}(\xi, \eta)\right\}, \quad \xi, \eta \in A .
$$

Assume that for some $x \in P$ there is no $x^{\prime} \in M$ such that dist $\left(\psi_{t}\left(x^{\prime}\right), \phi_{t}(x)\right) \leq \rho$, $t \in \mathbb{R}$. Then, given $s \in \mathbb{R}$ there is no $y \in M_{s}$ such that dist $\left(\phi_{t}\left(\phi_{s}(x)\right), \psi_{t}(y)\right) \leq \rho$ for $t \geq 0$, for, otherwise, if $\phi_{t_{n}}\left(\phi_{s}(x)\right) \rightarrow x, t_{n} \rightarrow \infty$, and $\psi_{t_{n}}(y)$ converges, say to $y_{\infty}$, we would have dist $\left(\phi_{t}(x), \psi_{t}\left(y_{\infty}\right)\right) \leq \rho, t \in \mathbb{R}$. Consequently, there is a $t^{*}>0$ such that if $y \in K_{\sigma^{\prime}}\left(x, t_{\nu}\right)$, then $\psi(y, t) \notin K_{\sigma^{\prime}}\left(x, t_{\nu}+t\right)$ for some $t, 0<t \leq t^{*}$ and any $\nu=1,2, \ldots$ Indeed, if such a $t^{*}$ did not exist, by taking limits we would find a positive $\psi$ semi-trajectory close to the positive $\phi$ semi-trajectory through $x$, which is absurd.

Choose $\nu>\nu_{0}(x)$ and such that $t_{\nu}+t^{*}<T$ and let $s(t)$ be a small positive continuous function such that for $t \in\left[0, t^{*}\right]$,

$$
\phi_{t}\left(h\left(s_{\nu}+s(t)\right)\right) \in \text { int } K_{\sigma^{\prime}}\left(x, t_{\nu}+t\right) .
$$

Since $s_{0}(t, u),\|u\|=1, \quad t_{\nu} \leq t \leq t_{\nu}+t^{*}$ is upper semicontinuous and $s_{1}(t, u)$, $0<s_{1}(t, u)<s_{0}(t, u)$, defined by

$$
\operatorname{dist}\left(\phi_{t}(x), \phi_{t}(g(s u))\right)>\rho^{\prime} \quad \text { if } s_{1}(t, u)<s \leq s_{0}(t, u),
$$

and

$$
\operatorname{dist}\left(\phi_{t}(x), \phi_{t}(g(s u))\right)=\rho^{\prime} \quad \text { if } s=s_{1}(t, u),
$$

is lower semicontinuous, it is easy to see that there exists a continuous $s^{\prime}(t, u)$, $s_{1}(t, u)<s^{\prime}(t, u)<s_{0}(t, u)$ defined for $\|u\|=1, t_{\nu} \leq t \leq t_{\nu}+t^{*}$. Let $C_{t}^{\prime}$ be defined by

$$
C_{t}^{\prime}=\left\{\phi_{t}(g(s u)):\|u\|=1,0 \leq s \leq s^{\prime}(t, u)\right\}
$$

for $t_{\nu} \leq t \leq t_{\nu}+t^{*}$.

For $y \in \chi_{\nu}$, let $t(y), 0 \leq t(y) \leq t^{*}$, be such that $\psi_{t}(y) \in$ int $K_{\sigma^{\prime}}\left(x, t_{\nu}+t\right)$ if $0 \leq t \leq t(y)$ and $\psi_{t}(y) \in \partial K_{\sigma^{\prime}}\left(x, t_{\nu}+t\right)$ if $t=t(y)$. It follows from (c) that $t(y)$ depends continuously on $y$ (see for instance [3, p. 198]). Now we define, for $i=0,1, \chi_{\nu}^{i} \subset \chi_{\nu}$ as the set of $y$ such that there exists a differentiable arc, contained in the interior of the ball of radius $\rho^{\prime}$ centred at $\phi\left(x, t_{\nu}+t(y)\right)$, transversal to $C_{t(y)}^{\prime}$ and joining 
$\psi(y, t(y))$ to $\phi_{t(y)}\left(h\left(s_{\nu}+s(t(y))\right)\right)$, with the property that its $(\bmod 2)$ intersection number with $C_{t(y)}^{\prime}$ is $i$. Since by (a) $\psi(y, t(y)) \in C_{t(y)}^{\prime}$ for no $y$ in $\chi_{\nu}$, and since two arcs joining $\psi(y, t(y))$ to $\phi_{t(y)}\left(h\left(s_{\nu}+s(t(y))\right)\right)$ contained in the above mentioned ball are homotopic within the ball, we conclude that $\chi_{\nu}^{i}$ is well defined, $i=0,1$, and that

$$
\chi_{\nu}^{0} \cup \chi_{\nu}^{1}=\chi_{\nu} \text { and } \chi_{\nu}^{0} \cap \chi_{\nu}^{1}=\varnothing .
$$

As both are open and since by (b) they are also non-empty, we have a contradiction. Thus, for each $x \in P$ there exists $x^{\prime} \in M$ such that dist $\left(\psi_{t}\left(x^{\prime}\right), \phi_{t}(x)\right) \leq \rho, t \in \mathbb{R}$. Now the proof of the proposition may be completed readily on the basis of the remarks included in the two last paragraphs at the end of the proof of proposition 3.1 in [3, p. 200].

The next corollary follows immediately from the previous proposition and well known results about stable and unstable manifolds for hyperbolic sets.

Corollary 2.5. Let $f$ be an expansive diffeomorphism of $M$. Assume that there is a collection $\mathscr{H}$ of transitive hyperbolic subsets of $M$ such that $\cup \mathscr{H}$ is dense in $\Omega$. If for each element of $\mathscr{H}$ the stable or unstable manifolds of its points are 1-dimensional, then $f$ is $\Omega$-persistent. This is true, in particular, when $\Omega$ contains a dense set of hyperbolic periodic points satisfying the assumptions regarding their stable or unstable manifolds.

COROLlARY 2.6. Let $f$ be an expansive $C^{2}$-diffeomorphism of $M$ and $\mu$ a normalized measure on the Borel sets of $M$ defined by a volume form, and invariant under $f$. Let $P$ be the subset of $M$ that consists of the points whose Lyapunov exponents are all different from zero. Assume that $\mu(P)>0$ and furthermore that for each $x \in P$ there is either only one positive exponent or only one negative exponent. Then $\bar{P}$ is persistent.

Proof. Since $P$ contains a dense subset of recurrent trajectories, proposition 2.1 applies by [5, proposition 4.7$]$.

The following lemma may be applied to get some other consequences of proposition 2.1 concerning low-dimensional $M$. Let $f$ be a diffeomorphism of the compact connected riemannian manifold $M$. A point $x \in M$ is stable if for every $\varepsilon>0$ there is $\delta>0$ such that if $y \in M$ and dist $(x, y)<\delta$, then dist $\left(f^{n}(x), f^{n}(y)\right) \leq \varepsilon$ for every $n \geq 0$.

LEMMA 2.7. If $f$ is expansive there are no stable points.

Proof. Suppose that $x \in M$ is stable, let $\varepsilon$ be less than half the expansivity constant of $f$ and let $\delta$ be as in the definition above; we assume $\delta<\varepsilon$. Let $(\hat{M}, \phi)$ be the suspension of $(M, f)$ and $\mathscr{U}, \dot{U}, \ddot{U}$ the Lyapunov functions for $\phi$. Let $\sigma>0$ be such that if we define $K_{\sigma}(x, t)$ as before, then dist $\left(\phi_{t}(x), \xi\right)<\delta$ for each $\xi \in K_{\sigma}(x, t)$. For $y \in \partial K_{\sigma}(x, 0)$ we have that dist $\left(f^{n}(y), f^{n}(x)\right)<\varepsilon, n \geq 0$ and therefore, on account of the expansivity of $f$ we must have that for some $T<0, \dot{u}\left(\phi_{t}(x), \xi\right)<0$ for every $\xi \in \partial K_{\sigma}(x, t)$ and every $t \leq T$. Indeed, if this were not the case we would reach a contradiction through a connectedness argument on an arc contained in $K_{\sigma}(x, t)$ and joining $\phi_{t}(x)$ to a point $\xi \in \partial K_{\sigma}(x, t)$ such that $\dot{u}\left(\phi_{t}(x), \xi\right)>0$. In this way we 
could find $y_{t} \in \partial K_{\sigma}(x, 0)$ such that $\phi_{s}(y) \in K_{\sigma}(x, s), t \leq s \leq 0$, for negative $t$ of arbitrarily large absolute value.

If $z \in M$ is an $\alpha$-limit point of $x$, we must then have that $\dot{u}\left(\phi_{t}(z), \eta\right) \leq 0$ for every $\eta \in \partial K_{\sigma}(z, t)$ and every $t \in \mathbb{R}$. This implies that if $\zeta \in K_{\sigma}\left(z, t_{0}\right)$ for some $t_{0}$, then $\phi_{t}(\zeta) \in K_{\sigma}(z, t)$ for any $t \geq t_{0}$; therefore $\phi(z, t) \in K_{\sigma}(x, 0)$ for arbitrarily large $t$. Since $\sigma$ may be chosen arbitrarily small, this implies $x \in \omega(z)$ and since if $\operatorname{dist}\left(x, x^{\prime}\right)<\delta$,

$$
\lim _{n \rightarrow \infty} \operatorname{dist}\left(f^{n}(x), f^{n}\left(x^{\prime}\right)\right)=0,
$$

this in turn, implies $x \in \omega(x)$. But if $y$ lies in a suitable neighbourhood $N \subset M$ of $x$, we have by the same reasoning, that $y \in \omega(y)=\omega(x)$. Now let $y^{\prime} \in \omega(x)$; then $y^{\prime} \in \omega(z)$, and therefore

$$
\lim _{n \rightarrow \infty}\left(f^{n}\left(f^{m}(z)\right), f^{n}\left(y^{\prime}\right)\right)=0
$$

for some $m>0$. It follows that for some $n, f^{n}\left(y^{\prime}\right)$ lies in $N$, i.e. that $\omega(x)$ is open. As $M$ is connected, $\omega(x)=M=\omega(z)$, but this implies that every point in $M$ is stable, which is absurd, since we could then find a sequence of iterates of $f$ converging to a trivial map, uniformly on $M$.

In the following theorems $\operatorname{dim} M=2$ or 3 .

THEOREM 2.8. Let $f$ be an expansive diffeomorphism of $M$. Assume that there is a collection of transitive hyperbolic subsets of $M$ whose union is dense in $\Omega$. Then $\Omega$ is persistent.

THEOREM 2.9. Let $f$ be an expansive $C^{2}$-diffeomorphism of $M$ and $\mu$ a normalized measure on the Borel sets of $M$, defined by a volume form and invariant under $f$. Assume that the subset $P$ of $M$ that consists of the points whose Lyapunov exponents are all different from zero has positive measure. Then $\bar{P}$ is persistent.

These theorems follow at once from lemma 2.7 and our previous results.

\section{Applications}

As may easily be seen the previous results apply equally well to the case of pseudo-Anosov 'diffeomorphisms' and to the case of the homeomorphisms considered in [4], in spite of the fact that they fail to be diffeomorphisms at a finite number of points (however, they are Lipschitzian homeomorphisms). They are expansive and have a dense set of hyperbolic periodic points (see [4] and [1, exposé 9, p. 177]). Therefore we may state:

Corollary 3.1. Pseudo-Anosov maps and the homeomorphisms constructed in [4] are persistent.

In order to find homeomorphisms conjugate to these we only need, according to the remark at the end of $\S 1$, to show that there are expansive homeomorphisms arbitrarily close to them, whose expansivity constants are bounded away from zero. 
This could be achieved by constructing suitable Lyapunov functions (see the remark after lemma 3.3 in [3]).

Let $f$ be a homeomorphism defined on an open and bounded neighbourhood $M$ of 0 in $R^{n}, f(0)=0$, and let $U: M \times M \rightarrow \mathbb{R}$ be a continuous function, $U(x, x)=0$. Let $N \subset M$ be a compact neighbourhood of 0 such that there exists a continuous function $\rho: N \rightarrow \mathbb{R}^{+}, \rho(x)>0$ if $x \neq 0$, with the following properties: $U(x, y)$ and $U(f(x), f(y))$ are defined if $x, y \in N$ and

$$
\begin{gathered}
\bar{U}(x, y)=U(f(x), f(y))-U(x, y) \geq 0 ; \\
\bar{U}(x, y) \geq r(x)\|x-y\|^{2},
\end{gathered}
$$

if $x \in N, y \in B_{x}(\rho(x))$. Here $B_{x}(\rho)$ denotes the ball with radius $\rho$ centred at $x$, and $r: N \rightarrow \mathbb{R}^{+}$is a continuous function such that $r(x)>0$ if $x \neq 0$. Let $V: M \times M \rightarrow \mathbb{R}$, $V(x, x)=0, x \in M$, be another continuous function such that

$$
|V(x, y)| \leq a\|x-y\|^{2}
$$

for some $a>0, x, y \in M$, with the property that $\bar{V}(x, y)>0$ if $x \neq y, x, y \in N$.

LEMMA 3.2. There exists a continuous function $W: M \times M \rightarrow \mathbb{R}, W(x, x)=0, x \in M$, and positive numbers $\rho_{0}, \rho_{1}, \delta, k, B_{0}\left(\rho_{1}\right) \subset$ int $N$, such that:

(i) $\bar{W}(x, y)>0 \quad$ if $x \neq y$ and $x \in N, y \in B_{x}(\delta)$;

(ii) for $x \in B_{0}\left(\rho_{0}\right)$ and $y \in B_{x}(\delta)$ we have $\bar{W}(x, y) \geq k \bar{V}(x, y)$;

(iii) for $\|x\| \geq \rho_{1}, W(x, y)=U(x, y), \bar{W}(x, y)=\bar{U}(x, y)$.

Proof. Let $\rho_{1}$ be such that $B_{x}\left(\rho_{1}\right), f\left(B_{x}\left(\rho_{1}\right)\right) \subset$ int $N$, and let $\rho_{1}^{\prime}<\rho_{1}$ be such that $f^{-1}\left(B_{x}\left(\rho_{1}^{\prime}\right)\right) \subset B_{x}\left(\rho_{1}\right)$. Choose $\rho_{0}^{\prime}<\frac{1}{2} \rho_{1}^{\prime}$, and $\rho_{0}<\rho_{0}^{\prime}$ such that $f\left(B_{0}\left(\rho_{0}\right)\right) \subset B_{0}\left(\rho_{0}^{\prime}\right)$ and let $c: \mathbb{R}^{n} \rightarrow \mathbb{R}^{+}$be a smooth function such that $c(x)=1$ if $x \in B_{0}\left(\rho_{0}^{\prime}\right)$ and $c(x)=0$ if $\|x\| \geq \rho_{1}^{\prime}$. Choose $\delta>0$ such that

and $k$ such that

$$
\delta<\min \left(\frac{1}{2} \rho_{1}, \min _{x \in N,\|x\| \geq \rho_{0}} \rho(x)\right)
$$

$$
\sigma=\min _{x \in N_{0}\|x\| \geq p_{0}} r(x) \geq 2 k a \sup _{x \in M}|c(f(x))-c(x)| .
$$

Let $W(x, y)=U(x, y)+k c(x) V(x, y)$. Then $W$ is defined in $M \times M$ and obviously $W(x, x)=0, x \in M$.

$$
\begin{aligned}
\bar{W}(x, y) & =\bar{U}(x, y)+k c(f(x)) V(f(x), f(y))-k c(x) V(x, y) \\
& =\bar{U}(x, y)+k c(f(x)) \bar{V}(x, y)+k(c(f(x))-c(f(y))) V(x, y)
\end{aligned}
$$

therefore if $\|x\| \leq \rho_{0}, \bar{W}(x, y)=\bar{U}(x, y)+k \bar{V}(x, y) \geq k \bar{V}(x, y)$ for every $y \in N$ and (ii) is proved.

On the other hand if $x \in N,\|x\| \geq \rho_{0}$,

$$
\begin{aligned}
\bar{W}(x, y) & \geq \sigma\|x-y\|^{2}-k a\left(\sup _{x \in M,\|x\| \geq \rho_{0}}|c(f(x))-c(x)|\right)\|x-y\|^{2} \\
& \geq \frac{1}{2} \sigma\|x-y\|^{2}
\end{aligned}
$$

if $\|x-y\| \leq \delta$, and this inequality, together with the previous one, proves (i). Since (iii) follows at once from the properties of $c$ and the choice of $\rho_{1}$ and $\rho_{1}^{\prime}$, this completes the proof. 
Now we consider, for $z$ in a neighbourhood of 0 in $\mathbb{C}$, the flow $\phi$ defined by the differential equation

$$
\dot{z}=\bar{z}^{p} / z^{p-1}=\bar{z}^{2 p-1} /|z|^{2 p-2},
$$

$p=\frac{1}{2} n, n=2,3, \ldots$, and prove

LEMMA 3.3. Let $V=\operatorname{Re}(z-\omega)\left(z^{2 p-1}-\omega^{2 p-1}\right)$; then

$$
\dot{V}(z, \omega) \geq|z-\omega|^{2}\left(|z|^{2 p-1}+|\omega|^{2 p-1}\right)(|z|+|\omega|)^{-1},
$$

where as before $\dot{V}(z, \omega)=\lim _{t \rightarrow 0}(1 / t)\left\{V\left(\phi_{t}(z), \phi_{t}(\omega)\right)-V(z, \omega)\right\}$.

Proof. $\dot{V}=A+B$, where

$$
A=\operatorname{Re}\left(\bar{z}^{p} / z^{p-1}-\bar{\omega}^{p} / \omega^{p-1}\right)\left(z^{2 p-1}-\omega^{2 p-1}\right)
$$

and

$$
B=(2 p-1) \operatorname{Re}(z-\omega)\left(z^{2 p-2} \bar{z}^{p} / z^{p-1}-\omega^{2 p-2} \bar{\omega}^{p} / \omega^{p-1}\right)
$$

Since

$$
A \geq \operatorname{Re}\left(|z|^{2 p}+|\omega|^{2 p}-\left(|\omega||z|^{2 p-1}+|z||\omega|^{2 p-1}\right)\right),
$$

and this expression is non-negative as may easily be shown, the proof will be complete if we show that

$$
B \geq|z-\omega|^{2}\left(|z|^{2 p-1}+|\omega|^{2 p-1}\right)(|z|+|\omega|)^{-1} .
$$

We have to show then, that

$$
\operatorname{Re}(z-\omega)\left(|z|^{r} \bar{z}-|\omega|^{r} \bar{\omega}\right) \geq|z-\omega|^{2}\left(|z|^{r+1}+|\omega|^{r+1}\right)(|z|+|\omega|)^{-1}
$$

where $r=2 p-2$. This inequality is equivalent to

$$
|z-\omega|^{2}\left(|z|^{r}|\omega|+|\omega|^{r}|z|\right)(|z|+|\omega|)^{-1}+\operatorname{Re}(z-\omega)\left(|z|^{r} \bar{\omega}-|\omega|^{r} \bar{z}\right) \geq 0,
$$

and except for non-negative factors, the left hand side equals

$$
\begin{aligned}
& \operatorname{Re}\left(\left(|z|^{r}-|\omega|^{r}\right)(|\omega| \bar{z}+|z| \bar{\omega})(z-\omega)\right) \\
& \quad=\left(|z|^{r}-|\omega|^{r}\right)(|z|-|\omega|)(|\omega||z|+\operatorname{Re} \bar{z} \omega)
\end{aligned}
$$

which is non-negative as we had to show.

LeMma 3.4. Let $f=\phi_{1}$. Then for some $K>0$,

$$
\bar{V}(z, \omega)=V(f(z), f(\omega))-V(z, \omega) \geq K|z-\omega|^{2}\left(|z|^{2 p-1}+|\omega|^{2 p-1}\right)(|z|+|\omega|)^{-1} .
$$

Proof. Since the right hand side of $\dot{z}=\bar{z}^{2 p-1} /|z|^{2 p-2}$ is Lipschitzian, there exist positive constants $h, k$, such that

$$
\left|\phi_{t}(z)-\phi_{t}(\omega)\right| \leq k|z-\omega|, \quad\left|\phi_{t}(z)-\phi_{t}(\omega)\right| \geq h|z-\omega|
$$

if $0 \leq t \leq 1$. Therefore, letting $P(z, \omega)=\left(|z|^{2 p-1}+|\omega|^{2 p-1}\right)(|z|+|\omega|)^{-1}$, we have that

$$
\begin{aligned}
\bar{V}(z, \omega) & =\int_{0}^{1} \dot{V}\left(\phi_{s}(z), \phi_{s}(\omega)\right) d s \\
& \geq \int_{0}^{1}\left|\phi_{s}(z)-\phi_{s}(\omega)\right|^{2} P\left(\phi_{s}(z), \phi_{s}(\omega)\right) d s \\
& =\left|\phi_{t}(z)-\phi_{t}(\omega)\right|^{2} P\left(\phi_{t}(z), \phi_{t}(\omega)\right)
\end{aligned}
$$


for some $t, 0 \leq t \leq 1$. As $\left|\phi_{t}(z)-\phi_{t}(\omega)\right|>h|z-\omega|$ and since

$$
k|z| \geq\left|\phi_{t}(z)\right| \geq h|z|, \quad k|\omega| \geq\left|\phi_{t}(\omega)\right| \geq h|\omega|,
$$

we can easily obtain the inequality in the statement of the lemma.

We again consider the diffeomorphism $f=\phi_{1}$, and assume that in some neighbourhood of 0 we have a Lyapunov function $W$ for $f$, constructed as in lemma 3.2 and therefore satisfying, for $x$ in some neighbourhood of 0 and $y$ such that dist $(x, y)<\delta$ for some fixed $\delta>0, \bar{W} \geq k \bar{V}$, wher: $V$ is the function of lemma 3.3. We also assume that

$$
W(x, y)=A_{11}\left(x_{1}-y_{1}\right)^{2}+A_{12}\left(x_{1}-y_{1}\right)\left(x_{2}-y_{2}\right)+A_{22}\left(x_{2}-y_{2}\right)^{2},
$$

where $x=\left(x_{1}, x_{2}\right), y=\left(y_{1}, y_{2}\right)$ and the $A_{i, j}=A_{i, j}(x, y)$ are functions of class $C^{2 p-2+\varepsilon}$, $\varepsilon>0$, such that at $x=0, y=0$, their $2 p-3$ jet is trivial. It follows from the lemmas that, for $x$ and $y$ satisfying the above conditions,

$$
\bar{W} \geq\left(\left(x_{1}-y_{1}\right)^{2}+\left(x_{2}-y_{2}\right)^{2}\right) H(x, y)
$$

where $H(x, y)$ is a positive definite homogeneous form of order $2 p-2$ in the variables $x_{1}, y_{1}, x_{2}, y_{2}$. Let $g$ be a homeomorphism close to $f$, and consider

$$
\begin{aligned}
\bar{W}_{g}(x, y) & =W(g(x), g(y))-W(x, y) \\
& =W(g(x), g(y))-W(f(x), f(y))+\bar{W}_{f}(x, y)
\end{aligned}
$$

we may show by considering the nature of $W$, that given $\rho>0$ such that $\left\{x \in \mathbb{R}^{2}\right.$ : $\|x\| \leq \rho\} \subset M$, there exist positive numbers $\varepsilon, \delta$ so that if $g(0)=0$ and $\left\|(g-f)^{\prime}(x)\right\| \leq \varepsilon$ for $\|x\| \leq \rho$, then $\bar{W}_{g}(x, y)>0, x \in B_{0}(\rho), 0<\|y-x\| \leq \delta$.

Now, let $f$ be a pseudo-Anosov map or a 'diffeomorphism' as those considered in [4] and let $\mathscr{A}=\left\{\left(A_{i}, \varphi_{i}\right): i=1, \ldots, n\right\}$ be a coordinate atlas for $M$. Given $\varepsilon>0$, we shall say that a homeomorphism $g: M \rightarrow M$ is $C^{1}-\varepsilon$-close to $f$ if for every $x \in M$, dist $(g(x), f(x))<\varepsilon$ and

$$
\left\|\left(\varphi_{j} \circ g \circ \varphi_{i}^{-1}-\varphi_{j} \circ f \circ \varphi_{i}^{-1}\right)^{\prime}\left(\varphi_{i}(x)\right)\right\|<\varepsilon
$$

for every $i$ such that $x \in A_{i}$, and every $j$ such that $f(x), g(x) \in A_{j}$. (If $\varepsilon$ is small enough there always exists such a $j$.) Now we may state:

THEOREM 3.5. Let $f: M \rightarrow M$ be a homeomorphism as those considered in [4] or a pseudo-Anosov map. There exists $\varepsilon>0$ such that if $g$ is a homeomorphism $C^{1}-\varepsilon-$ close to $f$ that coincides with $f$ at singular points, then $g$ is conjugate to $f$.

Proof. By the remark at the end of $\S 1$, corollary 3.1, and [3, lemma 3.3], we have only to show that there is a Lyapunov function $U$ and a $\delta>0$ such that for any $g$ satisfying the assumptions of the theorem we have $\bar{U}_{g}(x, y)>0$ if $x \in M$ and $0<\operatorname{dist}(x, y)<\delta$.

Consider one of the homeomorphisms of [4] or an iterate of a pseudo-Anosov map such that all its singularities are fixed points. Since at singular points there are coordinate neighbourhoods such that these 'diffeomorphisms', when expressed in those coordinates, coincide with the $f$ of lemma 3.4 (see [1], [2], [6]) we only need to show that there is a Lyapunov function $U^{0}$ (smooth except at singular points) 
such that for $y$ close to $x$,

$$
\bar{U}^{0}(x, y) \geq \rho(\operatorname{dist}(x, y))^{2} \quad \text { for some } \rho>0,
$$

provided $x$ lies outside some fixed neighbourhoods of the singular points, and that for $x$ inside these neighourhoods, it satisfies the assumptions of lemma 3.2 and condition (*). In fact once we have such a function we define (modulo coordinates), for $x$ inside the mentioned neighbourhoods, $U(x, y)$ as the $W(x, y)$ of lemma 3.2, ( $V$ being the function of lemma 3.3), and for $x$ outside some smaller neighbourhoods of the singular points, we define $U(x, y)$ as $U^{0}(x, y)$. By the remarks that follow the proof of lemma 3.4 it is easy to show that if $\varepsilon>0$ is small enough, there is a $\delta>0$ depending only on $\varepsilon$ such that $\bar{U}_{g}(x, y)>0$ for $x \in M, 0<\operatorname{dist}(x, y)<\delta$, provided $g$ satisfies for this $\varepsilon$ the hypothesis of the theorem.

Let $f$ be a homeomorphism like those considered in [4] obtained by lifting the Anosov diffeomorphism $h: T^{2} \rightarrow T^{2}$ through the projection $\pi: M \rightarrow T^{2}$. Let $u^{0}$ be a quadratic Lyapunov function for $h$ (see [3, p. 194]) and define $U^{0}(x, y)$ by $u^{0}(\pi(x), \pi(y))$; since, as may easily be shown, $U^{0}$ has the required properties, the proof is complete in this case.

Now let $f$ be a pseudo-Anosov 'diffeomorphism' and $m \in \mathbb{Z}^{+}$such that all the singularities of $f^{m}$ are fixed points and all their 'prongs' also remain fixed. We construct $U^{0}$ for $f^{m}$ in the following way: let $x$ and $y$ be nearby points in $M$; we consider the stable (unstable) leaf through $y$, find its intersection $z(\hat{z})$ with the unstable (stable) leaf through $x$, and take the transversal measure $\mu(x, y)(\hat{\mu}(x, y))$ of the segment $[x, z]$ (resp. $[x, \hat{z}])$. Let $U^{0}(x, y)=\mu^{2}(x, y)-\hat{\mu}^{2}(x, y)$; it is easy to check that this $U^{0}$ has all the required properties. Since if $g^{m}$ has expansivity constant $\alpha$ the same is true for $g$, the proof is complete.

\section{REFERENCES}

[1] A. Fathi, F. Laudenbach \& V. Poénaru. Travaux de Thurston sur les surfaces. (Seminaire Orsay). Asterisque, 66-67 (1979).

[2] M. Gerber \& A. Katok. Smooth models of Thurston's pseudo-Anosov maps. Preprint (1980).

[3] J. Lewowicz. Lyapunov functions and topological stability. J. Differential Equations (2) 38 (1980), 192-209.

[4] T. O'Brien \& W. Reddy. Each compact orientable surface of positive genus admits an expansive homeomorphism. Pacific J. Math. (3) 35 (1970), 737-741.

[5] Y. Pesin. Characteristic Lyapunov exponents and smooth ergodic theory. Russian Mathematical Surveys, (4) 32 (1977) pp. 747-817.

[6] W. Thurston. On the geometry and dynamics of diffeomorphisms of surfaces. Preprint.

[7] P. Walters. Anosov diffeomorphisms are topologically stable. Topology 9 (1970), 71-78. 